\title{
Antoine Culioli
}

(1924-2018)

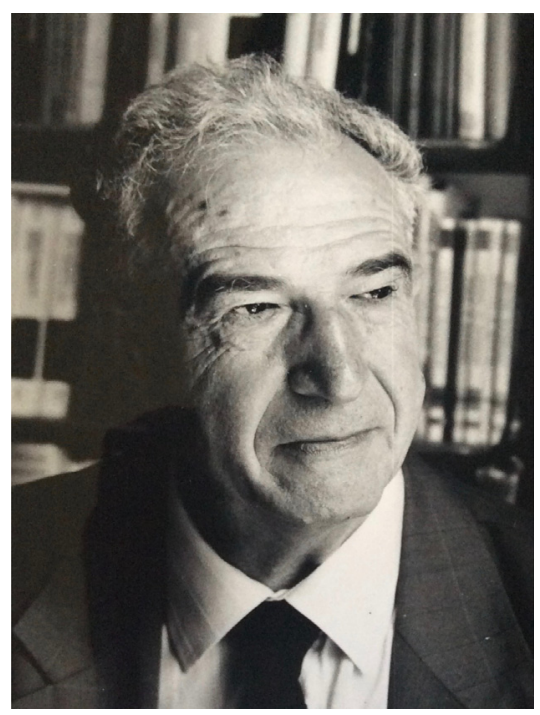

L'UFR de linguistique de l'Université Paris-Diderot a pour origine le département de recherche linguistique (DRL) fondé (1972) par Antoine Culioli qui nous a quittés le 9 février dernier. Il fut aussi l'un des pères fondateurs de notre université pluridisciplinaire et de son département d'anglais (1970). Ce que l'on sait moins c'est que le Laboratoire d'Histoire des Théories Linguistiques lui doit son nom et l'impulsion qui mena à sa fondation (1984).

Culioli voyait grand. Le DRL devait rassembler un ensemble de chercheurs venus d'horizons divers, réunis par l'idée que la linguistique est la science $d u$ langage construite sur la connaissance de la diversité des langues. Cette connaissance devait avoir un fondement conceptuel et une représentation formelle. Culioli avait également fondé un laboratoire CNRS, consacré à ce programme : il existe encore aujourd'hui, quoique transformé dans ses assises théoriques. 
Le département devint vite un centre d'accueil réputé qui, outre de nombreux invités étrangers, hébergeait souvent des enseignants chercheurs qui étaient en poste dans d'autres établissements mais participaient aux programmes ambitieux du département ${ }^{1}$. C'est ainsi qu'il accueillait J.-C. Chevalier, professeur à Paris 8 , qui était avec J. Stéfanini, d'Aix, l'un des seuls linguistes à offrir aux doctorants des séminaires d'histoire des sciences du langage. Quand les deux chercheurs recrutés par le CNRS (Sylvain Auroux en 1979 et Irène Rosier l'année d'après, ancienne élève de Culioli comme angliciste) souhaitèrent quitter le laboratoire lillois où ils avaient été affectés, il les accueillit également au DRL et poussa tout ce petit monde à présenter une demande de création d'un laboratoire CNRS que devait diriger J.-C. Chevalier. Ce n'était pas seulement quelques chercheurs CNRS qui allaient ainsi graviter autour du DRL, mais tout ce que la Société d'histoire et d'épistémologie des sciences du langage (créée en 1979 par S. Auroux, D. Droixhe et $\mathrm{C}$. Porset) pouvait fédérer de chercheurs internationaux. L'intérêt de Culioli pour l'histoire tenait du même élan que celui qu'il avait pour la philosophie (c'était un excellent connaisseur des Ideen de Husserl): la recherche d'un enracinement conceptuel solide. Il imposa le nom de «théorie» dans le titre du laboratoire pour exclure les historiens anecdotiques ou factuels. Nous étions parfaitement d'accord puisque c'est ce même souci qui nous avait poussés à introduire le terme d' «épistémologie» dans le nom de notre association et le titre de notre revue. «Épistémologie» lui paraissait trop galvaudé et sujet au bavardage philosophique (nous étions au début des années quatre-vingts et il y avait encore pléthore de personnalités enclines à faire un cours d' « épistémologie » sur Saussure en lieu et place d'un cours d'initiation à la linguistique). Ainsi, le DRL disposait non seulement d'un laboratoire CNRS en histoire, de chercheurs spécialisés, d'un séminaire de doctorat, mais Culioli avait fait introduire un module obligatoire d'histoire de la linguistique pour la maîtrise de linguistique formelle (module assuré au début par P. Bergheaud, S. Auroux, et I. Rosier). Le message général était l'impossibilité de construire une linguistique de haut niveau sans une connaissance historique poussée et théorique.

Culioli n'a pas seulement été notre mentor (aux côtés d'autres comme A. Joly, B. Pottier ou M. Gross), il est probablement l'un des plus grands linguistes français $\mathrm{du} \mathrm{xx}^{\mathrm{e}}$ siècle. Passé par la rue d'Ulm, il était agrégé d'anglais et docteur d'État avec une thèse de doctorat consacrée au moyen-anglais (Contribution à l'étude $d u$ subjonctif et de la coordination en moyen-anglais, 1960). Mais c'est à la linguistique, conçue comme une science qui aborde le langage au travers de la diversité des langues, plutôt qu'à la philologie, qu'il consacre enseignement

1 Le Laboratoire d'informatique linguistique dirigé par Maurice Gros contribuait aussi largement à la réputation de Paris 7. Culioli avait lui-même créé un laboratoire CNRS de linguistique formelle qu'il dirigeait. 
et séminaires. Orateur exceptionnel et perpétuel collectionneur d'exemples linguistiques (en toutes langues) qui ouvrent la réflexion à la perplexité, il a peu écrit. Ce travailleur infatigable a su développer une théorie cohérente basée sur les opérations énonciatives, c'est-à-dire des activités dûment répertoriées qui permettent aux êtres humains de communiquer.

Il est évidemment l'héritier de G. Guillaume et d'É. Benveniste. Mais les opérations énonciatives ne sont pas de mystérieuses opérations mentales. Ce sont des représentations formelles dont on retrouve la trace dans l'énoncé, trace ellemême prise dans un réseau d'oppositions. Un énoncé est toujours un résultat et il n'est tel que par l'énonciation. Autrement dit les «entités» linguistiques ne perdurent pas en elles-mêmes. Je pense que c'est par cette recherche d'opérations sous-jacentes que Culioli peut à la fois dépasser en finesse les grammaires catégorielles et aborder la diversité des phénomènes présents dans la diversité des langues. C'est exactement l'inverse d'une approche par le lambda-calcul où l'on peut travailler en donnant un nom aux entités, donc en présupposant leur existence comme une boite noire. C'est aussi abandonner la vieille théorie représentationnelle qui remonte à la théorie aristotélicienne du signe. Il n'y a pas d'universel (d'éléments subsumant la totalité d'une certaine diversité) mais des "invariants langagiers» qui subsistent comme tels sous une diversité de transformations. Quand on lui parlait des conséquences philosophiques de ses positions, Culioli vous regardait avec ce clignement d'œil qui doit avoir quelque chose de corse et que j'avais maintes fois affronté chez mon maître Desanti ; autrement dit on s'était perdu dans un bavardage de philosophes. C'est J.-P. Desclés qui a poussé le plus loin la mathématisation des théories culioliennes quoique son penchant pour les grammaires catégorielles l'en ait quelque peu éloigné. Je me souviens de mon émerveillement lorsque j'ai lu sa démonstration selon laquelle on pouvait obtenir des énoncés accusatifs ou ergatifs en enchaînant différemment le même fonds d'opérations. On s'en doutait dès que l'on admettait la possibilité d'une traduction, mais personne ne peut résister à la beauté d'une démonstration formelle.

Cette vision a le défaut de ses qualités: si l'on parvient aisément (parfois de façon lumineuse) à définir les opérations et leurs résultats (comme l'opération prédicative ou l'opération de repérage), l'objet sur lequel elles portent («notion» ou « domaine notionnel ») est quelque chose de bien moins clair au nominaliste que je suis. Par ailleurs on ne dispose pas, que je sache, de principe d'Occam susceptible de limiter le nombre des opérations pour expliquer la grande variété des oppositions que l'on rencontre dans la diversité des langues. L'une des difficultés que n'ont pas évitée certains disciples tardifs est une sophistication qui se perd dans une infinité de détails en rencontrant dans telle ou telle particule de telle langue des nuances dont il peut sembler que le locuteur n'a que faire. 
Antoine Culioli a eu des centaines de disciples dans le monde entier qui ont développé ses conceptions. Celles-ci se retrouvent dans la structuration qu'il avait voulue pour le DRL où il avait rassemblé théoriciens de toutes obédiences, spécialistes de quantité de langues (notamment non-indo-européennes), mathématiciens, informaticiens, historiens des sciences du langage. Il pratiquait luimême l'interdisciplinarité dans un séminaire commun avec le psychologue $\mathrm{F}$. Bresson et le logicien suisse J.-B. Grize (spécialistes des logiques dites naturelles). Les étudiants avaient donné au groupe le nom de « $\mathrm{BCG} »$ qui était celui du célèbre vaccin (obligatoire) contre la tuberculose. Organisateur visionnaire et convivial, Culioli avait su faire du DRL un lieu créatif où sont passés souvent dans l'enthousiasme et l'amitié, parfois dans la fureur et la dissidence, pour enseigner ou se former, la plupart des acteurs de la vie scientifique des sciences du langage de la fin du $\mathrm{xx}^{\mathrm{e}}$ siècle. Partisan d'une formation par la recherche, il a aussi su créer ou attirer au DRL de nombreux laboratoires associés au CNRS. Après sa retraite il a longtemps tenu un séminaire très fréquenté à l'ENS de la rue d'Ulm. Il a beaucoup enseigné et peu écrit, toujours sous la pression de ses disciples. Si, malgré tout, de nombreux textes de lui ont circulé, cela a souvent été dans des feuilles confidentielles publiées au DRL. Le lecteur est cependant aujourd'hui en mesure de comprendre l'étendue de son travail.

Sylvain Auroux

\section{Références}

Culioli, Antoine, 1990-1999. Pour une linguistique de l'énonciation, 3 vols, Paris, Orphys.

- 1995. Cognition and Representation in Linguistic Theory, Amsterdam, John Benjamins.

- 2014. L'arco e la freccia. Scritti scelti, Bologne, Il Mulino.

Ducard, Dominique \& Normand, Claudine(dir.), 2006. Antoine Culioli, Un homme dans le langage, Paris, Ophrys.

Kouame, Kan Frédéric (2017). Comprendre la linguistique d'Antoine Culioli, Paris, l'Auteur. 